\title{
Small Resolutions of Schubert Varieties and Kazhdan-Lusztig Polynomials
}

\author{
By \\ Parameswaran SANKARAN* and P. VANCHINATHAN*
}

\section{$\S 1$. Introduction}

Let $G$ be a complex semisimple algebraic group. Let $B, T, W$, and $S$ denote respectively a Borel subgroup, a fixed maximal torus contained in $B$, the Weyl group of $G$ with respect to $T$, and the set of fundamental reflections in $W$. Let $P$ denote a parabolic subgroup which contains $B$. We denote by $W_{P} \subset W$ the Weyl group of $P$. For $\lambda \in W / W_{p}$ let $e_{\lambda}$ denote the corresponding $T$-fixed point of $G / P$. Let $X(\lambda) \subset G / P$ denote the Schubert subvariety determined by $\lambda \in W / W_{p}$. Thus, $X(\lambda)$ is the Zariski closure of the $B$-orbit of $e_{\lambda} \in G / P$. The point $e_{\lambda}$ will be referred to as 'the centre' of $X(\lambda)$.

Recall that a resolution $p: \tilde{X} \rightarrow X$ of an irreducible complex projective variety $X$ is said to be small if, for each $i>0$ one has

$$
\operatorname{codim}_{X}\left\{x \in X \mid \operatorname{dim} p^{-1}(x) \geq i\right\}>2 i \text {. }
$$

If $p: \tilde{X} \rightarrow X$ is a small resolution, then for the intersection cohomology sheaf $\mathscr{K}^{\prime}(X)$ (with respect to the middle perversity), the stalk $\mathscr{X}^{\prime}(X)$, is isomorphic to the singular cohomology group $H^{\prime}\left(p^{-1}(x) ; \mathbb{C}\right)$ for any $\mathrm{i} \geq 0$. Suppose $p: \tilde{X}(\lambda) \rightarrow X(\lambda)$ is a small resolution of Schubert variety $X(\lambda) \subset G / B, \lambda \in W$, and let $X(\tau) \subset X(\lambda)$ be a Schubert subvariety. It is well-known that the Poincaré polynomial in $q^{1 / 2}$ of the fibre $p^{-1}\left(e_{\tau}\right)$ of $p$ is equal to the Kazhdan-Lusztig polynomial $P_{\tau, \lambda}(q)$, where $\tau \leq \lambda, \lambda, \tau \in W$ [4]. Suppose that, for some $g \in G$, $g X(\lambda)=X(\lambda)$. Then $g$ induces an isomorphism of the intersection cohomology sheaf $\mathscr{H}(X)$ such that $\mathscr{H}(X)$, maps onto $\mathscr{H}(X)_{q_{1}}$. In particular $P_{\theta, \lambda}(q)=P_{\tau, \lambda}(q)$ if $P_{\lambda} X(\theta)=X(\tau) \subset X(\lambda)$ where $P_{\lambda}$ denotes the largest parabolic subgroup that stabilizes $X(\lambda)$.

Suppose $X(\lambda) \subset G / P$. Let $\lambda_{0} \in W$ denote the representative of $\lambda$ of maximal length. Then $P_{\lambda_{v}}=P_{\lambda}$, the largest parabolic that stabilizes $X(\lambda)$. Also if $X(\tau) \subset X(\lambda)$ is $P_{\lambda}$-stable, then so is $X\left(\tau_{0}\right) \subset X\left(\lambda_{0}\right)$.

Communicated by M. Kashiwara, January 15, 1994.

1991 Mathematics Subject Classifications: 14M15.

* School of Mathematics, SPIC Science Foundation, 92, G.N. Chetty Road, Madras 600017 , INDIA. 
In our previous paper [6], we exhibited small resolutions for certain Schubert varieties in $G / P_{n}$ where $G=S O(2 n)$ or $S p(2 n)$. Here $P_{n}$ denotes the parabolic subgroup which corresponds to omitting the right end root $\alpha_{n}$, where we follow the conventions of [1] for labelling the simple roots of $G$. We also exhibited Schubert varieties in $S p(2 n) / P_{n}$ for which there exists no small resolution at all. In Theorem 1 of the present paper we shall give an inductive formula for the Poincaré polynomial of the fibres when $p: \tilde{X}(\lambda) \rightarrow X(\lambda)$ is a small resolution constructed in [6]. This readily yields a formula for the Kazhdan-Lusztig polynomial $P_{\tau, \lambda_{0}}(q), \tau \leq \lambda_{0}$, where $\lambda_{0}$ denotes the representative of $\lambda \in W / W_{p_{n}}$ of maximal length in $W$. See Theorem 2.4, [6]. In view of our remarks in the previous paragraphs, we need only consider $P_{\lambda}$-stable subvarieties of $X(\lambda)$ while computing Kazhdan-Lusztig polynomials.

A similar formula was obtained in the case of Grassmannian Schubert varieties by A.V. Zelevinskii. In fact our proof of Theorem 1 is similar to that given in [7] for Grassmannian Schubert varieties. Using Theorem 1 we obtain the following result. In order to emphasise the dependence of $P_{\tau, \lambda}$ for $\tau, \lambda \in W$, $\tau \leq \lambda$, on $(W, S)$, we write $P_{\tau, \lambda}^{W}$ for the Kazhdan-Lusztig polynomial.

Theorem. Let $W$ be the Weyl group of $G=S O(2 n)$ or $\operatorname{Sp}(2 n)$. Let $\lambda \in W$ be such that $X(\lambda)$ admits a small resolution given by Corollary 4.3 [6]. Let $\theta \leq \lambda$. Then there exist elements $\theta^{\prime}, \lambda^{\prime}$ in $W^{\prime}=W(S L(M))$ for a suitable integer $M$, such that

$$
P_{\theta, \lambda}^{W}=P_{\theta^{\prime}, \lambda^{\prime}}^{W^{\prime}}
$$

For the precise statement see Theorem 2. The above theorem reduces calculation of Kazhdan-Lusztig polynomials of certain pairs of elements $\tau \leq \lambda$ in the Weyl group of $G=S p(2 n)$ or $S O(2 n)$ to the calculation of such polynomials for a suitable pair of elements in the Weyl group of $S L(M)$ for some $M$. We do not know if the above theorem holds without any restriction on $\lambda \in W$. The above theorem can be interpreted as comparing the nature of the singularity at $e_{\theta}$ in $X(\lambda) \subset G / B$ with that of the singularity at $e_{\theta^{\prime}}$ in $X\left(\lambda^{\prime}\right) \subset S L(M) / B$. However the above theorem should be deducible as a consequence of a statement about the geometry of singularities of the Schubert varieties involved. The precise formulation of such a theorem is not clear to us.

We shall also consider the homogeneous variety $E_{6} / P_{6}$. Some results on small resolutions, and description of singular loci for most of the Schubert varieties in $E_{6} / P_{6}$ are obtained. In cases where we obtain small resolutions, we determine the Poincaré polynomials of the fibres of the resolution. We are able to completely determine which Schubert varieties in $E_{6} / P_{6}$ are smooth. It turns out that smooth Schubert varieties are all homogeneous varieties of the form $P / Q$ where $P$ and $Q$ are suitable parabolic subgroups of $E_{6}$. The singular locus of a Schubert variety in $E_{6} / P_{6}$ is determined using the following observation: 
Fact. Let $p: \tilde{X} \rightarrow X$ be a desingularization of an irreducible normal complex variety. Let $Z=\left\{x \in X \mid \operatorname{dim} p^{-1}(x) \geq 1\right\}$. Suppose that $p^{-1}(Z)$ has codimension at least 2 . Then $Z$ is the singular locus of $X$.

The reader is referred to Lemma 4.4 [5] for a proof.

It follows easily from the above fact that if $p: \tilde{X} \rightarrow X$ is a small resolution of a normal irreducible variety, then the singular locus of $X$ is equal to $\left\{x \in X \mid \operatorname{dim} p^{-1}(x) \geq 1\right\}$. In particular if $X(\lambda) \subset G / B$ admits a small resolution, then $x \in X(\lambda)$ is rationally smooth point if and only if $x$ is a smooth point of $X(\lambda)$. Here $G$ can be any semisimple complex algebraic group. It is known that for Schubert varieties in $S L(n) / B$, rational smoothness of a point is equivalent to smoothness [2]. However it is not known if every Schubert variety in $S L(n) / B$ admits a small resolution.

\section{Acknowledgments}

The authors are grateful to Professors C.S. Seshadri and V. Lakshmibai for many valuable comments.

\section{§2. Bott-Samelson Resolutions}

In [6], we considered Bott-Samelson type resolutions $p: \tilde{X}(\lambda) \rightarrow X(\lambda)$ of Schubert varieties in $G / P$ where $G$ is a complex semisimple algebraic group and $P$ a parabolic subgroup of $G$. It was shown that certain Schubert varieties in $S p(2 n) / P_{n}$ and $S O(2 n) / P_{n}$ admit small resolutions of the Bott-Samelson type. We calculate here the Poincaré polynomials of the fibres of $p: \tilde{X}(\lambda) \rightarrow X(\lambda)$. For this purpose we first give an alternative, more convenient description of $p: \tilde{X}(\lambda) \rightarrow X(\lambda)$.

We consider the case $G=S O(2 n)$ first. The case of $S p(2 n)$ can be handled similarly and hence will not be discussed in detail.

2.1. Let $V$ be a 2 -dimensional complex vector space with 'standard basis' $\left\{e_{1}, \ldots, e_{2 n}\right\}$. Let $\beta: V \times V \rightarrow C$ be the symmetric bilinear form defined by $\beta\left(e_{t}, e_{j}\right)=\delta_{t, 2 n+1-1}, 1 \leq i \leq j \leq 2 n$. The matrix of $\beta$ with respect to the standard basis is $\left(\begin{array}{ll}0 & J \\ J & 0\end{array}\right)$ where $J$ is the $n \times n$ matrix $\left(J_{l \jmath}\right), J_{l \jmath}=\delta_{\imath, n+1-\jmath}$. Let $V^{\imath}=\left\langle e_{1}, \ldots, e_{\imath}\right\rangle$ $:=\operatorname{span}\left\{e_{1}, \ldots, e_{l}\right\}, 1 \leq i \leq 2 n$. Note that $\left(V^{t}\right)^{\perp}=V^{2 n-t}, 1 \leq i \leq n$. Thus we have the "standard flag"

$$
\mathscr{F}: 0=V^{0} \subset V^{1} \subset \cdots \subset V^{n} \subset\left(V^{n-1}\right)^{\perp} \subset \cdots \subset\left(V^{1}\right)^{\perp} \subset V .
$$

Note that $\beta\left(V^{n}, V^{n}\right)=0$.

By definition, $S O(2 n)$ is the group of all $\mathbb{C}$-linear automorphisms of $V$ having determinant 1 which preserve the bilinear form $\beta$. Let $B$ be the subgroup of 
$S O(2 n)$ such that $B$ fixes the standard flag $\mathscr{F} . B$ is a Borel subgroup of $S O(2 n)$. Let $T$ be the subgroup of all linear automorphisms of $V$ such that $e_{t} \mapsto t_{1} e_{t}$, $t_{t} t_{2 n+1-l}=1,1 \leq i \leq 2 n, t_{t} \in \mathbb{C}^{*}$. Then $T$ is a maximal torus of $S O(2 n)$ and $T \subset B$. Let $P$ be the parabolic subgroup of $S O(2 n)$ which fixes the component $V^{n}$ of the standard flag $\mathscr{F}$. Then $P \supset B$ and in fact $P=P_{n}$, the parabolic subgroup which corresponds to omitting the right end root $\alpha_{n}$ (with respect to this choice of $T$ ). $S O(2 n) / P_{n}$ can now be identified with that component of $\{W \subset V \mid \beta(W, W)=0$, $\operatorname{dim} W=n\}$ which contains $V^{n}$. In fact this is just the variety.

$$
\left\{W \subset V \mid \beta(W, W)=0, \operatorname{dim} W=n, n-\operatorname{dim} W \cap V^{n} \text { is even }\right\} .
$$

2.2. Now the $T$ fixed points of the space $S O(2 n) / P_{n}$ are $e_{\lambda}=\left\langle e_{\lambda_{1}}, \ldots, e_{\lambda_{n}}\right\rangle$ where $\lambda=\left(\lambda_{1}, \ldots, \lambda_{n}\right)$ satisfies (i) $1 \leq \lambda_{1}<\cdots<\lambda_{n} \leq 2 n$, (ii) if $k$ occurs in $\lambda$, then $2 n+1-k$ does not occur in $\lambda$, (iii) $n-r$ is even where $r=\#\left\{j \mid \lambda_{j} \leq n\right\}$.

Let $I_{n, 1}$ denote the set of all sequence $\lambda=\left(\lambda_{1}, \ldots, \lambda_{r}\right)$ of integers with $1 \leq \lambda_{1}<\cdots<\lambda_{1} \leq n$. Note that any sequence $\lambda_{1}, \ldots, \lambda_{1}$, with $1 \leq \lambda_{1}<\cdots<\lambda_{r} \leq n$ and $n-r$ even uniquely determines a sequence $\lambda=\left(\lambda_{1}, \ldots, \lambda_{n}\right)$ with $\lambda_{1+1}>n$ satisfying conditions (i)-(iii) (cf. [3]) above and hence a $T$-fixed point $e_{\lambda}$. Thus the $T$-fixed points of $S O(2 n) / P_{n}$ are labelled by $\bigcup_{n-\text { reven }} I_{n, l}$. One can identify $W / W_{P_{n}}$ with $\cup_{n-\text { even }} I_{n, r}$, where the Bruhat order is given by $\lambda \geq \mu$ if $\lambda \in I_{n, r}, \mu \in I_{n, s}, r \leq s$ and $\lambda_{1} \geq \mu_{1}, \ldots, \lambda_{1} \geq \mu_{1}$.

For $\lambda \in I_{n, r}, n-r$ even, the Schubert variety determined by $\lambda$ is the $B$-orbit closure of $e_{\lambda}$, namely,

$$
X(\lambda)=\left\{W \in S O(2 n) / P_{n} \mid \operatorname{dim} W \cap V^{\lambda_{1}} \geq i, 1 \leq i \leq r\right\} .
$$

2.3. Similarly we regard $S p(2 n)$ as the group of linear automorphisms of $V$ which preserve the skew-symmetric form $\beta^{\prime}$ whose matrix with respect to the standard basis is $\left(\begin{array}{cc}0 & J \\ -J & 0\end{array}\right)$.

The subgroups $B, T$ and $P_{n}$ are defined just as in the case of $S O(2 n)$ using the standard flag $\mathscr{F}$. We identify $\operatorname{Sp}(2 n) / P_{n}$ with $\left\{W \mid \beta^{\prime}(W, W)=0, \operatorname{dim} W=n\right\}$.

2.4. The Schubert varieties of $S p(2 n) / P_{n}$ are indexed by

$$
W / W_{p_{n}}=\bigcup_{0 \leq 1 \leq n} I_{n, l}, \text { where for } \lambda \in I_{n, l} .
$$

One has

$$
X(\lambda)=\left\{W \in \operatorname{Sp}(2 n) / P_{n} \mid \operatorname{dim} W \cap V^{\lambda_{1}} \geq i, 1 \leq i \leq n\right\}
$$

with centre $e_{\lambda}=\left\langle e_{\lambda_{1}}, \ldots, e_{\lambda_{n}}\right\rangle$. Here $\lambda_{t}$ for $r \leq i \leq n$ are determined by the following conditions [3]: (i) $\left(\lambda_{1}, \ldots, \lambda_{n}\right) \in I_{2 n n}$, (ii) if $\lambda_{t}$ occurs in $\lambda$, then $2 n+1-\lambda$, does not occur in $\lambda$, and (iii) $\lambda_{t+1}>n$. 
2.5. We recall the notations of [6]. For $\lambda=\left(\lambda_{1}, \ldots, \lambda_{1}\right) \in I_{n, r}$, a maximal subsequence of consecutive integers in $\lambda$ is referred to as a 'block' of $\lambda$. The sequence $\lambda$ is clearly the concatenation of its blocks. Let $a_{\imath}$ be the length and $k_{1}$ the last term of the $i$ th block. Then $\lambda$ determines a $2 \times m$ matrix $\left(\begin{array}{lll}k_{1} & \cdots & k_{m} \\ a_{1} & \cdots & a_{m}\end{array}\right)$. On the other hand starting with a $2 \times m$ matrix $\left(\begin{array}{lll}k_{1} & \cdots & k_{m} \\ a_{1} & \cdots & a_{m}\end{array}\right)$ with $0<k_{1}<\cdots$ $<k_{m} \leq n, 0<a_{t}<k_{t}-k_{t-1},\left(k_{0}=0\right), \sum a_{t}=r$, we obtain a unique element $\lambda$ of $I_{n, l}$ having at most $m$ blocks. We often write

$$
\lambda=\left(\begin{array}{lll}
k_{1} & \cdots & k_{m} \\
a_{1} & \cdots & a_{m}
\end{array}\right)
$$

2.6. Let $G=S O(2 n)$ or $S p(2 n)$. Suppose $\lambda=\left(\begin{array}{lll}k_{1} & \cdots & k_{m} \\ a_{1} & \cdots & a_{m}\end{array}\right) \in W / W_{P_{n}}$. Let $\mathscr{F}_{\lambda}$ denote the partial flag $0 \subset V^{h_{1}} \subset \cdots \subset V^{k_{m}} \subset V$. Then one can see easily that

$$
X(\lambda)=\left\{W \in G / P_{n} \mid \operatorname{dim} W \cap V^{h_{i}} \geq a_{1}+\cdots+a_{l}, 1 \leq i \leq m\right\} .
$$

Suppose $\mathscr{F}_{\lambda}^{\prime}$ is another flag

$$
0 \subset W^{h_{1}} \subset W^{h_{2}} \subset \cdots \subset W^{k_{m}} \subset \cdots \subset V
$$

with $\quad \beta\left(W^{k_{m}}, W^{h_{m}}\right)=0 \quad$ (resp. $\quad \beta^{\prime}\left(W^{h_{m}}, W^{h_{m}}\right)=0$ ) when $\quad G=S O(2 n) \quad$ (resp. $G=S p(2 n))$. Then

$$
X\left(\lambda, \mathscr{F}_{\lambda}^{\prime}\right)=\left\{W \in G / P_{n} \mid \operatorname{dim} W \cap W^{k,} \geq a_{1}+\cdots+a_{t}, 1 \leq i \leq m\right\}
$$

is isomorphic to the Schubert variety $X(\lambda)=X\left(\lambda, \mathscr{F}_{\lambda}\right)$.

2.7. We shall now give a slightly different description of the Bott-Samelson type resolution of $X(\lambda) \subset G / P_{n}$ constructed in Theorem 4.2 of [6] which is more useful for our present purposes. Let $\lambda$ equal either (i) $\left(\begin{array}{lll}k_{1} & \cdots & k_{m} \\ a_{1} & \cdots & a_{m}\end{array}\right)$ with exactly $m$ blocks, $G=S O(2 n), S p(2 n)$, or (ii) $\left(\begin{array}{llll}k_{1} & \cdots & k_{m} & n \\ a_{1} & \cdots & a_{m} & 1\end{array}\right)$ with exactly $m+1$ blocks, and $G=S O(2 n)$. Let $M$ denote the number of blocks in $\lambda$; thus $M=m$, or $m+1$. Let $k_{m+1}=n, a_{m+1}=1$.

Choose an $i, 0 \leq i<m$. Let

$$
\lambda^{\prime}=\left(\begin{array}{ccccccc}
k_{1} & \cdots & k_{t-1} & k_{t}+a_{t+1} & k_{t+2} & \cdots & k_{M} \\
a_{1} & \cdots & a_{t-1} & a_{t}+a_{t+1} & a_{t+2} & \cdots & a_{M}
\end{array}\right) .
$$

Then the number of blocks of $\lambda^{\prime}$ is $M-1$ except when $m=1$, in which case $\lambda^{\prime}$ is a smooth variety. Let $U$ be any vector subspace of $V$ such that $V^{h_{l}} \subset U \subset V^{h_{t+1}}$ 
and $\operatorname{dim} U=k_{l}+a_{t+1}$. The space of all such $U$ can be identified with the Grassmannian $G_{a_{t+1}}\left(V^{k_{t+1}} / V^{k_{t}}\right)$, where $U \mapsto \bar{U}$, the image of $U$ under the projection $V^{k_{t+1}} \rightarrow V^{k_{t+1}} / V^{k_{i}}$. Let $\mathscr{F}_{\lambda^{\prime}}(U)$ denote the partial flag

$$
0 \subset V^{k_{1}} \subset \cdots \subset V^{k_{1-1}} \subset U \subset V^{k_{t+2}} \subset \cdots \subset V^{k_{m}} \subset V .
$$

Let

$$
\begin{aligned}
X(\lambda ; i) & =\left\{(U, W) \mid \bar{U} \in G_{a_{t+1}}\left(V^{k_{t+1}} / V^{k_{\imath}}\right), U \supset V^{k_{\iota}}, W \in X\left(\lambda^{\prime} ; \mathscr{F}_{\lambda^{\prime}}(U)\right)\right\} \\
& \subset G_{a_{t+1}}\left(V^{k_{t+1}} / V^{k_{t}}\right) \times G / P_{n} .
\end{aligned}
$$

One can readily identify $X(\lambda ; i)$ with the associated fibre space $P_{\lambda} \times_{R} X\left(\lambda^{\prime}\right)$, $R=P_{\lambda} \cap P_{\lambda^{\prime}}$ where $P_{\lambda}$ denotes the parabolic which stabilizes the Schubert variety $X(\lambda)$. The second projection $G_{a_{t+1}}\left(V^{k_{t+1}} / V^{k_{l}}\right) \times G / P_{n} \rightarrow G / P_{n}$ maps $X(\lambda ; i)$ onto $X(\lambda)$. The map $\pi: X(\lambda ; i) \rightarrow X(\lambda)$ defined by the second projection map is surjective birational morphism.

The restriction to $X(\lambda ; i)$ of the first projection map is a locally trivial fibre bundle with fibre $X\left(\lambda^{\prime}\right)$. Iterating this procedure for $\lambda^{\prime}$ in the place of $\lambda$ finally leads to a desingularization $p: \tilde{X}(\lambda) \rightarrow X(\lambda)$. We remark that $\tilde{X}(\lambda)$ depends on the successive choice of ' $i$ ' made at each stage. Note that the definition of $X(\lambda)$ depends on the choice of the flag $\mathscr{F}_{\lambda}$. For emphasis we write $p_{\bar{\gamma}_{\lambda}}: \tilde{X}\left(\lambda ; \mathscr{F}_{\lambda}\right)$ $\rightarrow X\left(\lambda ; \mathscr{F}_{\lambda}\right)$, for the above resolution.

2.8. We note that $\tilde{X}\left(\lambda ; \mathscr{F}_{\lambda}\right)$ is a subvariety of a certain product $G / Q_{1} \times G / Q_{2} \times \cdots \times G / Q_{m+1}$ where the $Q_{1}$ are certain maximal parabolic subgroups of $G$. (For example, $Q_{1}=P_{k_{1}+a_{1+1}}$ ). One has the following compatibility properties:

(i). Suppose $g \in G$, and $\mathscr{F}^{\prime}=g \mathscr{F}: g V^{1} \subset \cdots g V^{n} \subset \cdots g V^{2 n}=V$. Then

$$
\begin{aligned}
& X\left(\lambda ; \mathscr{F}_{\lambda}{ }^{\prime}\right)=\left\{g W \mid W \in X\left(\lambda ; \mathscr{F}_{\lambda}\right)\right\} . \\
& \tilde{X}\left(\lambda ; \mathscr{F}_{\lambda}^{\prime}\right)=\left\{g x \mid x \in \tilde{X}\left(\lambda ; \mathscr{F}_{\lambda}\right)\right\},
\end{aligned}
$$

where by $g x$ we mean image of $x \in G / Q_{1} \times \cdots \times G / Q_{m+1}$ under the diagonal action.

(ii). Also,

$$
g p_{\overline{\lambda_{\lambda}}}(x)=p_{\overline{\lambda_{\lambda}^{\prime}}}(g x) \in X\left(\lambda ; \mathscr{F}^{\prime}\right) \text { for every } x \in \tilde{X}\left(\lambda ; \mathscr{F}_{\lambda}\right) .
$$

Furthermore,

$$
\tilde{X}\left(\lambda ; \mathscr{F}_{\lambda}\right)=\left\{(U, x) \mid \bar{U} \in G_{a_{t+1}}\left(V^{k_{t+1}} / V^{k_{t}}\right), U \supset V^{k_{\imath}}, x \in \tilde{X}\left(\lambda^{\prime} ; \mathscr{F}_{\lambda^{\prime}}(U)\right)\right\} .
$$

(iii). In particular, if $g \in P_{\lambda}$, then $g \mathscr{F}=\mathscr{F}$ and we deduce that $p_{\bar{T}}$ is $P_{\lambda}$ equivariant. If $x, y \in X(\lambda)$ are in the same $P_{\lambda}$-orbit then it follows that $p_{\bar{\zeta}}^{-1}(x)$ 
and $p_{\zeta}^{-1}(y)$ are isomorphic as varieties, Consequently they have the same Poincaré polynomial.

Note. The above description of $\tilde{X}(\lambda)$ parallels the one given by Zelevinskii for Grassmannian Schubert varieties [7].

\section{§3. Main Results and Proofs}

3.1. Let $G=S O(2 n)$ or $S p(2 n)$. Let $\lambda \in W / W_{P_{n}}$ equal either (i) $\left(\begin{array}{lll}k_{1} & \cdots & k_{m} \\ a_{1} & \cdots & a_{m}\end{array}\right)$ with exactly $m$ blocks, $G=S O(2 n), \quad S p(2 n), \quad$ or $\quad$ (ii) $\quad\left(\begin{array}{llll}k_{1} & \cdots & k_{m} & n \\ a_{1} & \cdots & a_{m} & 1\end{array}\right)$ and $G=S O(2 n)$.

We let

$$
b_{t-1}=k_{t}-k_{t-1}-a_{t}, 1 \leq i \leq m,\left(k_{0}=0\right),
$$

and $b_{m}=N-k_{m}$ where $N=n$ if $G=S O(2 n)$, and $N=n+1$ if $G=S p(2 n)$. (Note that in [6] $b_{m}$ was defined to be $N-k_{n}-\frac{1}{2}$ ). We denote the number of blocks in $\lambda$ by $M$, so that $M=m$, or $m+1$. It follows from Theorem 3.1 of [6] that the largest parabolic subgroup $P_{\lambda} \subset G$ which leaves $X(\lambda) \subset G / P_{n}$ stable corresponds to omitting the roots $\left\{\alpha_{k_{1}}, \cdots, \alpha_{k_{m}}\right\}$.

3.2. Let $X(\tau)$ be a $P_{\lambda}$-stable subvariety of $X(\lambda) \subset G / P_{n}$. Suppose that for $\tau \leq \lambda, c(\tau, \lambda)=\left(c_{1}, \cdots, c_{M}\right)$ is the 'depth' sequence of $\tau$ in $\lambda$ so that

$$
\tau=\left(\begin{array}{cccccc}
k_{1} & k_{2} & \cdots & k_{m} & \vdots & n \\
a_{1}+c_{1} & a_{2}+c_{2}-c_{1} & \cdots & a_{m}+c_{m}-c_{m-1} & \vdots & 1
\end{array}\right)
$$

where the column $\left(\begin{array}{l}n \\ 1\end{array}\right)$ is present precisely when $G=S O(2 n)$ and $c_{m}$ is odd. See Corollary 3.3 [6]. Now starting with a Schubert subvariety $X(\theta) \subset X(\lambda), \theta=$ $\left(\theta_{1}, \cdots, \theta_{1}\right) \in I_{n, 1}$, one has the following description of the Schubert variety $X(\tau) \subset X(\lambda)$ such that $\theta=X(\tau)=P_{\lambda} \cdot X(\theta)$ : Let $t_{t}=\#\left\{j \mid k_{t-1}<\theta_{J} \leq k_{t}, 1 \leq j \leq s\right\}$, $1 \leq i \leq m$, and let $\varepsilon=0$ or 1 according as $\#\left\{j \mid k_{m}<\theta_{1} \leq n, 1 \leq j \leq s\right\}$ is even or odd. Then

$$
\tau=\left(\begin{array}{ccccc}
k_{1} & \cdots & k_{m} & \vdots & n \\
t_{1} & \cdots & t_{m} & \vdots & \varepsilon
\end{array}\right)
$$


Since $X(\theta) \subset X(\lambda)$, it follows from 2.6 that $\sum_{1 \leq J \leq l} t_{j} \geq \sum_{1 \leq j \leq l} a_{j}$. We let $c_{l}=$ $\sum_{1 \leq j \leq l}\left(t_{j}-a_{J}\right), 1 \leq j \leq m$, and $c_{m+1}=c_{m}+\varepsilon-1$. Then the depth of $X(\tau)$ in $X(\lambda)$ is $c(\tau, \lambda)=\left(c_{1}, \cdots, c_{M}\right)$.

3.3. Assume that the following conditions on $\lambda$ hold:

(i) $k_{m}<N-a_{m}$,

(ii) for $i \geq 1$, one has $k_{m}<N-\left(a_{m}+a_{m-1}+\cdots+a_{t}\right)+\left(b_{m-1}+\cdots+b_{t}\right)$.

Under the above assumptions $X(\lambda)$ admits a Bott-Samelson type resolution $p: \tilde{X}(\lambda) \rightarrow X(\lambda)$ which is small. Indeed choose $i, 0 \leq i<m$ such that $b_{t} \leq a_{l}$, $\left(a_{0}=\infty\right)$ and $a_{t+1} \leq b_{t+1}$. Let $\lambda^{\prime}$ be as in 2.7. This leads to the first step of a BottSamelson type resolution. Then the above conditions hold for $\lambda^{\prime}$ and we iterate this procedure to obtain a Bott-Samelson type resolution. The resolution so obtained is small and is $P_{\lambda}$-equivariant (cf. Theorem 4.2, [6]). In particular, denoting the Poincare polynomial in $q^{1 / 2}$ of $p^{-1}\left(e_{\theta}\right)$ by $P_{\theta, \lambda}(q)$, one has, by 2.8 (iii) above, $P_{\theta, \lambda}(q)=P_{\tau, \lambda}(q)$.

We now state the main results of this paper: Let $\left[\begin{array}{l}a \\ b\end{array}\right]$ be the Gaussian binomial coefficient $\left(q^{a}-1\right) \cdots\left(q^{a-b+1}-1\right) /\left(q^{b}-1\right) \cdots(q-1)$. We follow the conventions that $\left[\begin{array}{l}a \\ b\end{array}\right]=0$ if either $b<0$ or $a<b$, and $\left[\begin{array}{l}0 \\ 0\end{array}\right]=1$.

Theorem 1. Let $G=S p(2 n)$ or $S O(2 n)$. Let $\lambda \in W / W_{P_{n}}$ satisfy 3.3(i) and 3.3(ii). With the above notations, one has the following inductive formula for the Poincaré polynomial of the fibre over $e_{\tau}$ of $p: \tilde{X}(\lambda) \rightarrow X(\lambda)$

$$
P_{\tau, \lambda}(q)=\sum_{d} q^{\left(c_{t}-d\right)\left(c_{t+1}-d\right)}\left[\begin{array}{c}
a_{t+1}-c_{t}+c_{t+1} \\
c_{t+1}-d
\end{array}\right]\left[\begin{array}{c}
b_{t}+c_{t}-c_{t+1} \\
c_{t}-d
\end{array}\right] P_{\tau(d), \lambda^{\prime}}(q),
$$

where $X(\tau(d))$ is the $P_{\lambda^{\prime}}$-stable subvariety of $X\left(\lambda^{\prime}\right)$ with depth sequence

$$
c\left(\tau(d), \lambda^{\prime}\right)=\left(c_{1}, \cdots, c_{t-1}, d, c_{t+2}, \cdots, c_{M}\right)
$$

3.4. As an immediate consequence we obtain the following theorem, which is a more precise restatement of the theorem stated in the introduction. Let $G=S O(2 n)$ or $S p(2 n)$. Let $\lambda, \tau \in W / W_{P_{n}}$ be as in the above theorem. Let $\lambda_{0}, \tau_{0} \in W$ denote the representatives of $\lambda, \tau \in W$ respectively of maximal length. Let $\Lambda=\left(\begin{array}{cccc}k_{1} & \cdots & k_{m} & N+c_{m} \\ a_{1} & \cdots & a_{m} & c_{m}\end{array}\right)$, where $N=n$ or $n+1$ according as $G=S O(2 n)$ or $S p(2 n) . \Lambda$ defines a Grassmannian Schubert variety $X^{G \prime}(\Lambda) \subset S L\left(N+c_{m}\right) / P_{1+c_{m}}$, where $r=\sum_{1 \leq i \leq m} a_{l}$. Let $X^{G \prime}(T)$ be the $P_{\Lambda}$-stable Schubert subvariety of $X^{G \prime}(\Lambda)$ with depth sequence $\left(c_{1}, c_{2}, \cdots, c_{m}, 0\right)$. Let $\Lambda_{0}, T_{0} \in W\left(S L\left(N+c_{m}\right)\right)$ denote the representatives of $\Lambda, T$ of maximal length respectively. We denote by $P_{v, w}^{w}$, the 
Kazhdan-Lusztig polynomial of the Weyl group $W$ defined by the pair $y, w \in W$, $y \leq w$. Then,

Theorem 2. Let $G=S O(2 n)$ or $S p(2 n)$. Let $W$ be the Weyl group G. Let $\lambda$, be as in Theorem 1. Let $\theta \in W$ be any element such that $\theta \leq \lambda_{0}$. Let $\bar{\theta} \in W / W_{P_{n}}$ denote its projection. Let $X(\tau)=P_{\lambda} \cdot X(\bar{\theta}) \subset X(\lambda)$. With the above notations,

$$
P_{\theta, \lambda_{0}}^{W}=P_{\tau_{0}, \lambda_{0}}^{W}=P_{T_{0}, \Lambda_{0}}^{W^{\prime}}
$$

where $W^{\prime}=W\left(S L\left(N+c_{m}\right)\right)$, the Weyl group of $S L\left(N+c_{m}\right)$.

Proof of Theorem 1. The proof is based on counting the $\mathbb{F}_{q}$-rational points on $p^{-1}\left(e_{\tau}\right)$ and an induction argument on $m$.

First note that $p$ is the composition

$$
\tilde{X}(\lambda) \stackrel{q}{\longrightarrow} X(\lambda, i) \stackrel{\pi}{\longrightarrow} X(\lambda),
$$

where $q(U ; x)=\left(U, p_{\tau_{(U)}}^{\prime}(x)\right), \quad p_{\bar{J}_{(U)}}^{\prime}$ being the inductively constructed small resolution

$$
p_{\zeta(U)}^{\prime}: \tilde{X}\left(\lambda^{\prime} ; \mathscr{F}(U)\right) \rightarrow X\left(\lambda^{\prime} ; \tilde{F}(U)\right)
$$

Thus

$$
\begin{aligned}
& p^{-1}\left(e_{\tau}\right)=\left\{(U, x) \mid \bar{U} \in G_{a_{t+1}}\left(V^{k_{t+1}} / V^{h_{\iota}}\right), U \supset V^{h_{\iota}}, p_{\bar{\zeta}(U)}^{\prime}(x)=e_{\tau}\right\} \\
& =\left\{(U, x) \mid \bar{U} \in G_{a_{\imath+1}}\left(V^{k_{1+1}} / V^{h_{\imath}}\right), U \supset V^{h_{\iota}}, x \in\left(p_{\bar{\zeta}(U)}^{\prime}\right)^{-1}\left(e_{\tau}\right)\right\} \\
& =\left\{(U, x) \mid \bar{U} \in G_{a_{t+1}}\left(V^{h_{t+1}} / V^{h_{\imath}}\right), U \supset V^{h_{i}}, x \in\left(p_{\bar{\zeta}(U)}^{\prime}\right)^{-1}\left(e_{\tau}\right)\right. \text {, } \\
& \left.\operatorname{dim} U \cap e_{\tau} \geq a_{1}+\cdots+a_{t+1}\right\} \\
& =\amalg F_{d} \text {, }
\end{aligned}
$$

where

$$
\begin{gathered}
F_{d}=\left\{(U, x) \mid x \in\left(p_{\zeta_{(U)}}^{\prime}\right)^{-1}\left(e_{\tau}\right), \bar{U} \in G_{a_{t+1}}\left(V^{k_{t+1}} / V^{k_{t}}\right), U \supset V^{k_{t}},\right. \\
\left.\operatorname{dim} U \cap e_{\tau}=a_{1}+\cdots+a_{t+1}+d\right\} .
\end{gathered}
$$

Therefore the number of $\mathbb{F}_{q}$-rational points in $F_{d}$ is equal to the product of the number of $\mathbb{F}_{q}$-rational points of the variety

$$
A_{d}=\left\{U \mid \bar{U} \in G_{a_{t+1}}\left(V^{h_{t+1}} / V^{h_{1}}\right), U \supset V^{h_{1}}, \operatorname{dim} U \cap e_{\tau}=a_{1}+\cdots a_{t+1}+d\right\}
$$

times the number of $\mathbb{F}_{q}$-rational points of the variety $\left(p_{\zeta(U)}^{\prime}\right)^{-1}\left(e_{\tau}\right)$.

For any two $U_{1}, U_{2} \in A_{d},\left(p_{\tau\left(U_{1}\right)}^{\prime}\right)^{-1}\left(e_{\tau}\right)$ are isomorphic and the number of $\mathbb{F}_{q}$-rational points of the variety $\left(p_{\zeta(U)}^{\prime}\right)^{-1}\left(e_{\tau}\right)$ equals the polynomial $P_{\tau(d), \lambda^{\prime}}$ where 
$\tau(d)$ is the $P_{\lambda^{\prime}}$-stable subvariety with depth sequence $\left(c_{1}, \cdots, c_{t-1}, d, c_{t+2}, \cdots, c_{m}\right)$. Therefore, one has the formula

$$
P_{\lambda, \tau}(q)=\sum_{d} N_{q}\left(A_{d}\right) P_{\tau(d), \lambda^{\prime}}(q)
$$

where $N_{q}\left(A_{d}\right)$ is the number of $\mathbb{F}_{q}$-rational points of the variety $A_{d}$.

To complete the proof, we need only show that $N_{q}\left(A_{d}\right)$ is as in the Theorem.

Let $E=e_{\tau}$, and let $E_{J}=V^{k_{\jmath}} \cap E$. Note that $V^{k_{l}} / E_{l} \hookrightarrow V^{k_{t+1}} / E_{t+1}$. For $U \in A_{d}$, one has

(i) $E_{t} \subset U \cap E \subset E_{t+1}$

(ii) $V^{k_{t}} / E_{\imath} \subset U / U \cap E \subset V^{k_{t+1}} / E_{l+1}$.

One has a morphism $\phi: A_{d} \rightarrow G_{1} \times G_{2}, \quad U \mapsto\left(U \cap E / E_{l}, U /\left(V^{k_{t}}+U \cap E\right)\right)$, where $G_{1}$ and $G_{2}$ are the grassmannians of $\left(a_{t+1}+d-c_{t}\right)$-spaces in $E_{t+1} / E_{t}$, and $\left(c_{l}-d\right)$-dimensional spaces in $\left(V^{k_{l}+1} / E_{t+1}\right) /\left(V^{k_{r}} / E_{l}\right) \cong V^{k_{t+1}} /\left(V^{k_{l}}+E_{l+1}\right)$, respectively. One shows that $\phi$ is a fibre bundle with fibre an affine space of dimension $\left(c_{t}-d\right)\left(c_{t+1}-d\right)$. It follows that

$$
N_{q}\left(A_{d}\right)=q^{\left(c_{t}-d\right)\left(c_{t+1}-d\right)}\left[\begin{array}{c}
a_{t+1}+c_{t+1}-c_{t} \\
a_{t+1}+d-c_{\imath}
\end{array}\right]\left[\begin{array}{c}
b_{t}-c_{t+1}+c_{t} \\
c_{t}-d
\end{array}\right] .
$$

As $\left[\begin{array}{l}a \\ b\end{array}\right]=\left[\begin{array}{c}a \\ a-b\end{array}\right]$, this completes the proof.

Proof of Theorem 2. Let $p: \tilde{X}(\lambda) \rightarrow X(\lambda)$ denote the Bott-Samelson type small resolution corresponding to the choice $\left(i, i^{\prime}, \ldots, i^{(m)}\right)$ (see 3.3). Thus, $0 \leq i<m, b_{t} \leq a_{\imath}, a_{t+1} \leq b_{t+1}$, and

$$
\lambda^{\prime}=\left(\begin{array}{ccccccc}
k_{1} & \cdots & k_{t-1} & k_{t}+a_{t+1} & k_{t+2} & \cdots & k_{M} \\
a_{1} & \cdots & a_{t-1} & a_{t}+a_{t+1} & a_{t+2} & \cdots & a_{M}
\end{array}\right)
$$

yields the first step of the small resolution $p$. Let $X(\tau)$ have depth sequence $\left(c_{1}, \ldots, c_{M}\right)$. Now, for any positive integer $c$,

$$
\Lambda=\left(\begin{array}{cccc}
k_{1} & \cdots & k_{m} & N+c \\
a_{1} & \cdots & a_{m} & c
\end{array}\right),
$$

and defines a Grassmannian Schubert variety

$$
X^{G \prime}(\Lambda) \subset S L(N+c) / P_{1+c}, \quad r=\sum a_{\jmath} .
$$

Note that for the same choice of $i$,

$$
\Lambda=\left(\begin{array}{ccccccc}
k_{1} & \cdots & k_{t-1} & k_{t}+a_{t+1} & \cdots & k_{m} & N+c_{m} \\
a_{1} & \cdots & a_{t-1} & a_{t}+a_{t+1} & \cdots & a_{m} & c_{m}
\end{array}\right)
$$


yields the first step of a small resolution constructed by Zelevinskii. By induction, one concludes that the same sequence of induces $\left(i, i^{\prime}, \ldots, i^{(m)}\right)$ yields a small resolution for the Grassmannian Schubert variety $X^{G r}(\Lambda)$. In particular we let $c=c_{m}$, and consider the $P_{\Lambda}$-stable Schubert subvariety $X^{G r}(T) \subset X^{G r}(\Lambda)$ which has 'depth' sequence $\left(c_{1}, \ldots, c_{m}, 0\right)$.

It is now immediate from Theorem 1 above, and Theorem 2 of Zelevinskii [7] and standard facts (see Theorem 2.4 of [6] and [4]) that

$$
P_{\theta, \lambda_{0}}^{W}=P_{T_{0}, \Lambda_{0}}^{W^{\prime}}
$$

Example 3. Take $G=S O(24) . W=W(S O(24))$ is a subgroup $S_{24}$. Let $\lambda=\left(\begin{array}{ll}6 & 8 \\ 3 & 1\end{array}\right) \in W / W_{P_{12}}$. Note that $\lambda$ satisfies conditions $3.3(\mathrm{i})$, (ii) and hence admits a small resolution. Then $\lambda_{0}=(24,23,22,18,16,15,14,13,8,6,5,4 \mid \cdots) \in W$ (cf. [3]). Take $\theta=(5,6,8,13,21,16,14,7,1,10,3,2 \mid \cdots) \in W$. Then $\theta \leq \lambda_{0}$. We shall compute $P_{\theta, \lambda_{0}}$ using our methods. First note that, denoting by $\bar{\theta}$ the coset in $W / W_{P_{n}}$ determined by $\theta$, one has

$$
\bar{\theta}=(1,2,3,5,6,7,8,10) .
$$

Now $P_{\lambda}$, the stabilizer of $X(\lambda) \subset S O(24) / P_{12}$, is the parabolic which omits the simple roots $\alpha_{6}$ and $\alpha_{8}$.

The $P_{\lambda}$-saturation of $X(\theta)$ is $X(\tau)$ where

$$
\tau=\left(\begin{array}{ccc}
6 & 8 & 12 \\
5 & 2 & 1
\end{array}\right)
$$

See 3.2. Therefore the Kazhdan-Lusztig polynomial $P_{\theta \cdot \lambda_{0}}(q)=P_{t}\left(p_{\tilde{X}}^{-1}\left(e_{\tau}\right)\right)$, the Poincaré polynomial of the fibre over $e_{\tau}$ for small resolution $p: \tilde{X}(\lambda) \rightarrow X(\lambda)$ (with $q=t^{2}$ ).

Now for $\lambda=\left(\begin{array}{ll}6 & 8 \\ 3 & 1\end{array}\right)$, one has $\left(k_{1}, k_{2}\right)=(6,8),\left(a_{1}, a_{2}\right)=(3,1),\left(b_{0}, b_{1}\right)=(3,1)$. Therefore the choice of $i=1$ leads to the first step of a small resolution of $X(\lambda)$. When $i=1, \lambda^{\prime}=\left(\begin{array}{l}7 \\ 4\end{array}\right)$. One then takes $\lambda^{\prime \prime}=\left(\begin{array}{l}4 \\ 4\end{array}\right) \cong S O(16) / P_{16}$ which is a smooth
variety.

Now $c(\tau, \lambda)=\left(c_{1}, c_{2}\right)=(2,3)$. Since $i=1$, the possible non-zero terms in the formula for $P_{t}\left(p^{-1}\left(e_{\tau}\right)\right)$ are when $d=0,1$, or 2 . Again $b_{2}-c_{2}+c_{1}=0$, and the only non-zero term corresponds to $d=c_{1}=2$.

Thus 


$$
\begin{aligned}
P_{\tau, \lambda}(q) & =q^{0}\left[\begin{array}{c}
1+3-2 \\
1
\end{array}\right] \cdot\left[\begin{array}{l}
0 \\
0
\end{array}\right] \cdot P_{\tau(2), \lambda^{\prime}}(q) \\
& =(1+q) \cdot P_{\tau(2), \lambda^{\prime}}(q)
\end{aligned}
$$

Now $\tau(2)=\left(\begin{array}{l}7 \\ 6\end{array}\right)$, and again using our formula

$$
P_{\tau(2), \lambda^{\prime}}(q)=q^{0}\left[\begin{array}{c}
4+2-0 \\
2
\end{array}\right] \cdot 1=\left[\begin{array}{l}
6 \\
2
\end{array}\right]=\left(1+q^{2}+q^{4}\right)\left(1+q+q^{2}+q^{3}+q^{4}\right)
$$

Thus, by substitution

$$
P_{\theta \cdot \lambda_{0}}(q)=P_{\tau, \lambda}(q)=(1+q)\left(1+q^{2}+q^{4}\right)\left(1+q+q^{2}+a^{3}+q^{4}\right) .
$$

\section{$\S$ 4. Schubert Varieties $\mathrm{im} \quad E_{6} / P_{6}$}

In this section we consider the Schubert subvarieties in $E_{6} / P_{6}$ and address the question of existence of Bott-Samelson type resolutions which are small. Here $P_{6}$ denotes the maximal parabolic which omits the root $\alpha_{6}$ (see Figure 1). We shall determine the singular loci for most of the Schubert subvarieties. Although our methods are applicable to other exceptional groups and other parabolic subgroups as well, we consider only $E_{6} / P_{6}$ because in this case the computations are manageable. First recall that the Dynkin diagram for the group $E_{6}$ is

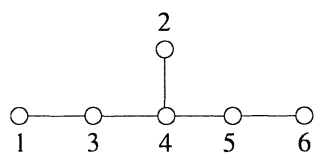

Figure 1

The numbers represent the simple roots of $E_{6}$.

4.1 One has the following coloured directed graph in which the set of Schubert varieties in $E_{6} / P_{6}$ form the vertices. The zero dimensional variety is at the bottom; vertices on the same horizontal represent varieties of the same dimension. There is an edge from $X(\lambda)$ to $X(\omega)$ with colour $i$ if and only if $s_{\alpha_{1}} \lambda=\omega, \lambda<\omega$ under the Bruhat order. Here $s_{\alpha_{t}} \in S$ denotes the fundamental reflection corresponding to the simple root $\alpha_{l}$. Reduced expressions for the minimal lift of $X(\lambda)$ in $E_{6} / B$ are obtained by simply reading off the colours of downward paths from $X(\lambda)$ to $X($ id $)$. 


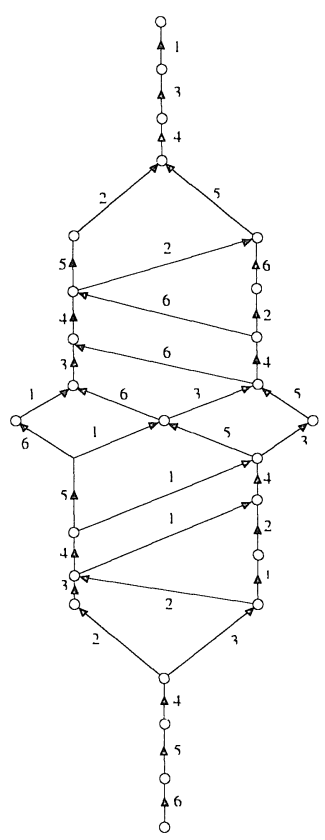

Figure 2

Lemma 4. The largest parabolic subgroup $P_{\lambda}$ which stabilizes a given Schubert variety $X(\lambda)$ corresponds to omitting those simple roots $\alpha_{1}$ such that $i$ is the colour of an edge which originates from $X(\lambda)$.

Proof. It is clear that $P_{\lambda}$ omits the simple roots which are labels of edges originating from $X(\lambda)$. If $s_{\alpha}$ is a fundamental reflection which is not the colour of any edge originating from $X(\lambda)$ one has $/_{6}\left(s_{\alpha} \lambda\right) \leq /_{6}(\lambda)$ where $/_{6}$ is the length function on $W / W_{P_{6}}$. From this it follows that $P_{\lambda}$ contains a representative for $s_{\alpha}$. Hence the lemma.

Lemma 5. Let $X(\tau) \subset X(\lambda)$. Then $X(\tau)$ is a $P_{\lambda}$ stable subvariety if and only if $P_{\lambda}$ omits $\alpha_{1}$ for every colour $i$ of an edge that originates at the vertex $X(\tau)$.

Proof. Immediate from the above lemma.

4.2. Our conventions for labelling the Schubert varieties are as follows: $X\left(\lambda_{1}\right)$ denotes the only Schubert variety of dimension $i$ when $i=0,1,2,3,13,14,15$, 16. When $i=4, \ldots, 12, j=1$, (resp. $j=2), X\left(\lambda_{t,}\right)$ denotes the Schubert variety of dimension $i$ which is a vertex on the left (resp. right) hand side of the above graph. The $X\left(\lambda_{8,0}\right)$ denotes the variety of dimension 8 at the 'centre' of the graph. 
Theorem 6. The smooth Schubert subvarieties in $E_{6} / P_{6}$ are $X\left(\lambda_{0}\right) \cong\left\{e_{t d}\right\}$, $X\left(\lambda_{1}\right) \cong \mathbb{P}^{1}, \quad X\left(\lambda_{2}\right) \cong \mathbb{P}^{2}, X\left(\lambda_{3}\right) \cong \mathbb{P}^{3}, X\left(\lambda_{4,1}\right) \cong X\left(\lambda_{4,2}\right) \cong \mathbb{P}^{4}, X\left(\lambda_{5,2}\right) \cong \mathbb{P}^{5}, \quad X\left(\lambda_{8,1}\right)$ $\cong S O(10) / P_{1}$.

Proof. That the above are the only possible smooth ones follow by a simple argument using Poincaré duality. We shall only show that $X:=X\left(\lambda_{8,1}\right) \cong S O(10) / P_{1}$, the remaining cases being similar. First note that the largest parabolic $P$ that leaves $X$ stable is that which omits the root $\left\{\alpha_{1}\right\}$ and that $X$ has no subvariety properly contained in $X$ which is stable under $P$. Thus $X$ is the $P$ orbit of $e_{t d}$. It follows that $X \cong P / Q$, where $Q$ is the isotropy at $e_{t d}$ for the $P$ action on $X$. Thus $Q=P \cap P_{6}$. By considering the Levi decomposition of $P$, one can show easily that $P$ contains a subgroup $G$ of type $D_{5}$ such that $R:=G \cap Q=G \cap P_{6}$ is the parabolic subgroup of $G$ which corresponds to omitting the left end root and that $G / R \cong P / Q \cong X$. Clearly, $G / R \cong S O(10) / P_{1}$ where the $P_{1} \subset S O(10)$ here stands for the parabolic subgroup of $S O(10)$ that corresponds to omitting the left end root of $S O(10)$. This completes the proof.

4.3. We shall now consider other Schubert varieties in $E_{6} / P_{6}$ and determine in most of the cases the singular loci. We shall exhibit small resolutions $p: \tilde{X}(\lambda) \rightarrow X(\lambda)$ for some of the Schubert varieties and calculate the Poincaré polynomials of the fibres over the $T$-fixed points.

We shall use the following abbreviations in the statements of our results. $\lambda$ always stands for the Schubert variety $X(\lambda)$ being considered. For a subset $J$ of $\{1, \ldots 6\}$ let $P_{J}$ denote the parabolic which corresponds to omitting the simple roots $\left\{\alpha_{\imath} \mid i \notin J\right\}$. The set $I \subset\{1,2,3,4,5,6\}$ denotes the set for which the parabolic subgroup $P_{I}=P_{\lambda}$, which is the parabolic that stabilizes $X(\lambda)$.

Recall the $\mathbb{F A C T}$ noted in the introduction.

Definition 7. If $p: \tilde{X} \rightarrow X$ satisfies the hypothesis of the $\mathbb{F} \mathbb{C} \mathbb{T}$, we call p a strict resolution.

Thus if $p$ is a small resolution, then it is strict.

Observe that $P_{\lambda}$ acts on $X(\lambda)$ and the smooth locus of $X(\lambda)$ contains the orbit of the centre $e_{\lambda}$ of $X(\lambda)$. It follows that the singular locus of $X(\lambda)$ is contained in a union of lower dimensional $P_{\lambda}$ orbits. We will make use of this observation in determining the singular locus of $X(\lambda)$. When $p: \tilde{X}(\lambda) \rightarrow X(\lambda)$ is a small resolution the Poincaré polynomial of the fibre over $e_{\tau}$ is the same as the Poincaré polynomial of the stalk at $e_{\tau}$ of the intersection cohomology sheaf of $X(\lambda)$. We compute the Poincaré polynomials of $p^{-1}\left(e_{\tau}\right)$ for each $P_{\lambda}$-stable subvariety $X(\tau) \subset X(\lambda)$. In view of the discussion in the introduction one can deduce the Poincaré polynomial of $p^{-1}\left(e_{\theta}\right)$ for any $X(\theta) \subset X(\lambda)$. We denote Poincaré polynomial (in the variable $q^{1 / 2}$ ) of $p^{-1}\left(e_{\tau}\right)$ by $K L_{t}$ or $K L_{l, \jmath}$ where 
$\tau=\lambda_{l}$ or $\tau=\lambda_{l, J}$ respectively, in the view of its relation to the Kazhdan-Lusztig polynomial.

\section{Theorem 8 .}

1. $\lambda_{5,1}: I=\{2,3,5,6\}$. Singular locus is $X\left(\lambda_{2}\right)$. The map $p: P_{\{3\}} \times_{B} X\left(\lambda_{4,1}\right) \rightarrow X(\lambda)$ is a small resolution. $K L_{2}=1+q$.

2. $\lambda_{6,1}: I=\{2,3,4,6\}$. Singular locus is $X\left(\lambda_{1}\right)$.

3. $\lambda_{6,2}: I=\{1,2,3,5,6\}$. Singular locus is $X\left(\lambda_{2}\right)$. The map $p: P_{\{2\}} \times_{B} X\left(\lambda_{5,2}\right) \rightarrow$ $X(\lambda)$ is a small resolution. $K L_{2}=1+q$.

4. $\lambda_{7,1}: I=\{2,3,4,5\}$. Singular locus is $X\left(\lambda_{0}\right)$.

5. $\lambda_{7,2}: I=\{1,2,4,6\}$. Singular locus is $X\left(\lambda_{4,1}\right)$. The map $p: P_{\{2,4\}} \times_{R} X\left(\lambda_{5,2}\right) \rightarrow$ $X(\lambda)$, where $R=P_{\{4\}}$, is a strict resolution.

6. $\lambda_{8,0}: I=\{1,2,4,5\}$. Singular locus is $X\left(\lambda_{4,1}\right)$. The map $p: P_{\{2,4,5\}} \times_{R} X\left(\lambda_{5,2}\right) \rightarrow$ $X(\lambda)$, where $R=P_{\{4.5\}}$, is a strict resolution.

7. $\lambda_{8,2}: I=\{1,2,3,4,6\}$. Singular locus is $X\left(\lambda_{1}\right)$.

8. $\lambda_{9,1}: I=\{1,2,4,5,6\}$. Singular locus is $X\left(\lambda_{4.1}\right)$. The map $p: P_{\{1\}} \times_{B} X(\lambda) \rightarrow$ $X(\lambda)$ is a small resolution. $K L_{4,1}=1+q$.

9. $\lambda_{9,2}: I=\{1,2,3,5\}$. Singular locus is $X(\tau)$ where $\tau$ is one of $\lambda_{6,2}$, or $\lambda_{2}$ or $\lambda_{0}$.

10. $\lambda_{10,1}: I=\{1,2,3,5,6\}$. Singular locus is $X\left(\lambda_{6,2}\right)$. The map $p: P_{\{1,3\}} \times_{R} X\left(\lambda_{8,1}\right) \rightarrow$ $X(\lambda)$, where $R=P_{\{3\}}$, is a small resolution. $K L_{6.2}=1+q, K L_{2}=1+q+q^{2}$.

11. $\lambda_{10.2}: I=\{1,3,4,5\}$. Singular locus is $X\left(\lambda_{5.2}\right)$, or $X\left(\lambda_{0}\right)$.

12. $\lambda_{11,1}: I=\{1,3,4,6\}$. The map $p: P_{\{1,3,4\}} \times_{R} X\left(\lambda_{8,1}\right) \rightarrow X(\lambda), R=P_{\{3.4\}}$, is a small resolution. Singular locus is $X\left(\lambda_{8,2}\right) . K L_{8.2}=1+q, K L_{5.2}=1+q+q^{2}, K L_{1}=1$ $+q+q^{2}+q^{3}$.

13. $\lambda_{11,2}: I=\{1,2,3,4,5\}$. Singular locus is $X\left(\lambda_{0}\right)$.

14. $\lambda_{12.1}: I=\{1,3,4,5,6\}$. Singular locus is $X\left(\lambda_{5,2}\right)$. The map $p: P_{\{1.3 .4 .5\}} \times_{R} X\left(\lambda_{8.1}\right)$ $\rightarrow X(\lambda), R=P_{\{3,4,5\}}$ is a small resolution. $K L_{5,2}=1+q+q^{2}$.

15. $\lambda_{12,2}: I=\{1,2,3,4,6\}$. Singular locus is $X\left(\lambda_{8,3}\right)$ or $X\left(\lambda_{1}\right)$.

16. $\lambda_{13}: I=\{1,2,3,5,6\}$. Singular locus is $X\left(\lambda_{10,1}\right)$. The map $p: P_{\{2\}} \times_{B} P_{\{1,3,4,5\}}$ $\times_{R} X\left(\lambda_{8,1}\right) \rightarrow X(\lambda)$, where $R=P_{\{3,4,5\}}$ is a strict resolution .

17. $\lambda_{14}: I=\{1,2,4,5,6\}$. Singular locus is $X\left(\lambda_{9,1}\right)$ or $X\left(\lambda_{4,1}\right)$.

18. $\lambda_{15}: I=\{2,3,4,5,6\}$. Singular locus is $X\left(\lambda_{8.1}\right)$.

Note. When $\lambda=\lambda_{9.2}, \lambda_{10.2}, \lambda_{12.2}, \lambda_{14}$ the above theorem does not completely determine the singular locus.

Proof. We know that the singular locus of $X(\lambda)$ is a union of $P_{\lambda}$-stable Schubert subvarieties. This allows us to determine the singular locus of $X(\lambda)$ in case $\lambda=\lambda_{5,1}, \lambda_{6,1}, \lambda_{6,2}, \lambda_{7,1}, \lambda_{8,2}, \lambda_{9,1}, \lambda_{11,2}, \lambda_{12,1}, \lambda_{15}$. For example, $X\left(\lambda_{1}\right)$ is the only $P_{\lambda_{82}}$-stable subvariety of $X\left(\lambda_{8,2}\right)$ of smaller dimension. As $X\left(\lambda_{8,2}\right)$ is not smooth one sees that $X\left(\lambda_{1}\right)$ is its singular locus. The same argument shows, for example, that the singular locus of $X\left(\lambda_{9,2}\right)$ is either $X\left(\lambda_{6,2}\right)$ or $X\left(\lambda_{2}\right)$ or $X\left(\lambda_{0}\right)$. (Note: 
$\left.X\left(\lambda_{0}\right) \subset X\left(\lambda_{2}\right) \subset X\left(\lambda_{6.2}\right)\right)$. To prove the rest of the theorem we proceed as follows: Let $P \supset Q$ be parabolic subgroups of a complex semisimple group $G$. The dimension of the projective variety $P / Q$ equals $\ell\left(\omega_{0}(P)\right)-\ell\left(\omega_{0}(Q)\right)$, where $\omega_{0}(P)$ denotes the longest element of the Weyl group of $P$ and $\ell$ denotes the length function. Using this and Lemma 2.1 of [6], one veritfies that the map $p$ is small or strict as asserted in each case. Using the $\mathbb{F A \mathbb { T }}$ mentioned in the introduction one determines the singular locus whenever $p$ is a strict resolution. We shall now show how to compute the Poincaré polynomials of fibres over $T$ fixed points. We shall only prove that when $\lambda=\lambda_{11,1}$, one has $K L_{5,2}=1+q+q^{2}$, others being similar. Note that $X\left(\lambda_{5,2}\right)$ is a $P_{\lambda}$-stable subvariety. Let $e$ denote the centre of $X\left(\lambda_{5,2}\right)$. One sees that $p^{-1}\left(X\left(\lambda_{5,2}\right)\right)=P \times_{R} X\left(\lambda_{4,2}\right)$, where $P=P_{\{1,3,4\}}$ and $R=P_{\{3,4\}}$. We wish to determine the fibre $F:=p^{-1}(e)$ of the map $p: P \times_{R} X\left(\lambda_{4,2}\right) \rightarrow X\left(\lambda_{5,2}\right)$. Consider the commutative diagram:

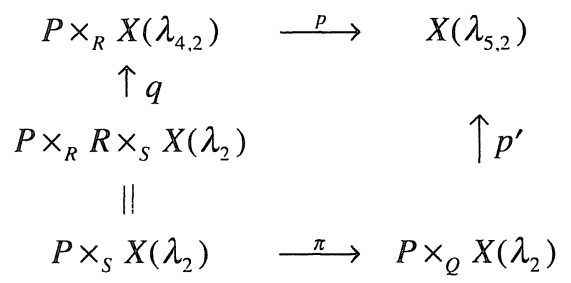

where $S=P_{\{3\}}, Q=P_{\{1,3\}}$. The morphisms $p^{\prime}$ and $q$ are induced by the respective multiplication maps, and $\pi$ is the projection. One has, for $g \in P, x \in R \times{ }_{S} X\left(\lambda_{2}\right)$, $q([g, x])=\left[g, q^{\prime}(x)\right]$ where $q^{\prime}$ is the map $q^{\prime}: R \times_{S} X\left(\lambda_{2}\right) \rightarrow X\left(\lambda_{4.2}\right)$ induced by multiplication. The map $q^{\prime}$ is birational. The map $q \mid q^{-1}(F): q^{-1}(F) \rightarrow F$ is an isomorphism. On the other hand $p^{\prime}$ is birational, and the map $\pi$ is a smooth bundle projection with fibre $Q / S \cong \mathbb{P}^{2}$. Let $\varepsilon \in P \times{ }_{Q} X\left(\lambda_{2}\right)$ be the point which is mapped to $e$ by $p^{\prime}$. Then

$$
p^{-1}(e)=F \cong q^{-1}(F)=\pi^{-1}(\varepsilon) \cong \mathbb{P}^{2} .
$$

Therefore $K L_{2}=$ Poincaré polynomial of $\mathbb{P}^{2}$ in $q^{1 / 2}=1+q+q^{2}$.

\section{Referemces}

[1] Bourbaki, N., Groupes et algèbre de Lie, Chapitres IV-VI, Hermann, Pairs (1968).

[2] Deodhar, V., Local Poincaré duality and nonsingularity of Schubert varieties, Comm. Alg., $\mathbb{1 3}$ (1985), 1379-1388.

[3] Lakshmibai, V. and Seshadri, C.S., Geometry of G/P-II, Proc. Ind. Acad. Sci., 87A (1978), 1-54.

[4] Kazhdan, D. and Lusztig, G., Schubert varieties and Poincaré duality, Proc. Symp. Pure Math., 36 (1980), 185-203.

[5] Narasimhan, M.S. and Ramanan, S., Moduli of vector bundles on a compact Riemann surface, Ann. Math., 89 (1969), 14-51.

[6] Sankaran, P. and Vanchinathan, P., Small resolutions of Schubert varieties in Symplectic and orthogonal Grassmannians, to appear in Publ. RIMS, 30 (1994).

[7] Zelevinskii, A.V., Small resolutions of singularities of Schubert varieties, Funct. Anal. Appl., 17 (2) (1983), 142-144. 